\title{
Entorno virtual 3D de la tabla periódica como apoyo en la educación de la química.
}

\author{
Sebastián Posada Calderón ${ }^{1}$, Yohana Monsalve Suárez ${ }^{1}$, Sandra Patricia Mateus Santiago ${ }^{2}$ \\ 1 Ingeniero Informático, Bancolombia, Carrera 52 \#14-30 Edificio COH Medellín. Correo electrónico: \\ posada.096@gmail.com,monsalveyoha@gmail.com \\ ${ }^{2}$ Doctora en Ingeniería-Sistemas, Profesora Titular, Politécnico Colombiano Jaime Isaza Cadavid, Carrera 48 \\ \# 7-151 Medellín. Correo electrónico: spmateus@elpoli.edu.co
}

\section{RESUMEN}

Este artículo plantea el desarrollo de un entorno virtual de la tabla periódica con el propósito de adquirir una herramienta que apoye el aprendizaje de la química, usando técnicas de realidad virtual. Para el desarrollo de esta propuesta, primero, se realizó la caracterización de los diferentes conceptos de la tabla periódica y lo concerniente a Realidad Virtual; luego, se diseñaron los componentes de la arquitectura, se modeló la base de datos y se realizaron los mockups; a partir de esto, se implementó el prototipo de la tabla periódica a través de técnicas de realidad virtual y modelado 3D y finalmente, se validaron los resultados del entorno virtual mediante un grupo de control de estudiantes de la materia química orgánica del Politécnico Jaime Isaza Cadavid, profesores y personas externas y se hizo la retroalimentación del entorno, con base en la encuestas realizadas.

Palabras clave: Entorno Virtual, Realidad Virtual, Tabla Periódica, Química, Modelado 3D.

Recibido: 23 de Julio de 2019. Aceptado: 04 de Diciembre de 2019.

Received: July 23, 2019. Accepted: December 04, 2019.

\section{D Virtual Environment of the periodic table as a support in the education of chemistry.}

\begin{abstract}
This paper proposes the development of a virtual environment of periodic table to acquire a tool as a support the learning of chemistry, using virtual reality techniques. first, the characterization of the different concepts of the periodic table and that concerning Virtual Reality was carried out, then, the architecture components were designed, the database was modeled and the mockups were made, from this, the prototype of the periodic table was implemented through virtual reality techniques and 3D modeling and finally, through a control group of students rolled in organic chemistry course in Politecnico Jaime Isaza Cadavid, teachers, and other people the results of the virtual environment were validated, after that, the feedback was made based on the surveys
\end{abstract}

Keywords: Virtual Environment, Virtual Reality, Periodic Table, Chemistry.

Como citar este articulo: S. Posada, Y. Monsalve, S. Mateus. "Entorno virtual 3D de la tabla periódica como apoyo en la educación de la química.", Revista Politécnica, vol. 15, no.30, pp.41-54, 2019. DOI:10.33571/rpolitec.v15n30a5 


\section{INTRODUCCIÓN}

El concepto de Realidad Virtual se concibe como una realidad simulada, que se construye con sistemas informáticos mediante el uso de formatos digitales. La construcción y visualización de esta realidad alternativa, requiere hardware y software lo suficientemente potente como para crear una experiencia inmersiva realista [1]. Por otro lado, la química es importante en la educación, porque se puede entender, cómo los principios químicos operan en todos los aspectos de la vida [2], sin embargo, los átomos y la cantidad de sustancias, pueden resultar demasiado abstractos y con ayuda de la Realidad Virtual los estudiantes pueden entender de una manera más interactiva los conceptos [3].

Adicionalmente, Torres [4] indica que la enseñanza y el aprendizaje de la Química exigen un nivel elevado de abstracción y preparación conceptual, que le permiten al estudiante, enfrentarse a la solución de problemas específicos, por lo tanto, es necesario la adopción de nuevas estrategias para la asimilación de los conceptos de Química, además, denota que, las nuevas tecnologías pueden ser usadas en el proceso educativo, equipando a cada estudiante con herramientas tecnológicas que faciliten la enseñanza individualizada y de esta manera, complementar la formación académica.

Es por lo anterior, que se propone el desarrollo de un entorno virtual de la tabla periódica, en el cual el estudiante tenga una herramienta de Realidad Virtual de apoyo, con el fin de buscar nuevas estrategias pedagógicas que complementan la educación tradicional.

Este artículo está organizado de la siguiente manera: en la sección 2, se muestra el estado del arte; de las secciones 3 a la 6 , se detalla la caracterización, diseño, desarrollo y validación del entorno virtual; y finalmente, se exponen las Conclusiones y los Trabajos Futuros.

\section{ESTADO DEL ARTE}

A continuación, se exponen los principales antecedentes de este artículo.

\section{Realidad Virtual y Realidad Aumentada en la educación}

El-Mounayri et al., [10] implementaron un entorno de Realidad Virtual como herramienta educativa para el aprendizaje del funcionamiento de una máquina fresadora que sirve para cortar y pulir metales. Los autores indican que este, se diseñó para permitir a los estudiantes y capacitar a los profesionales sobre el uso de la máquina sin la necesidad de estar en un laboratorio físico, afirman que los usuarios operan el entorno virtual mediante un casco de realidad virtual y dispositivos de entrada estándar tal como un mouse o un teclado, en el entorno virtual se muestra un tutorial sobre como operar la máquina sin la necesidad de instrucciones externas, sin embargo, la herramienta requiere de un casco de Realidad Virtual de alto costo, lo que limita a la personas, acceder a esta herramienta.

Así mismo, Zhang et al., [11], resaltan la importancia de la Realidad Virtual para apoyar la educación de prácticas que pueden resultar peligrosas, si se les da un mal manejo. Los autores muestran el diseño e implementación de una realidad virtual de fuego y mecanismos para mitigar el peligro y aprendizaje del correcto manejo cuando se llegue a presentar un incendio en un campus. En esta misma vía, se encuentra la patente presentada por Peters, Postlethwaite, \& Wallace [12] donde evidencian que el aprendizaje en el mundo real puede resultar costoso, representar riesgos de seguridad y consumir mucho tiempo de un instructor; es por ello que, ellos plantean un subsistema programable, con un rastreador espacial, lo cual es una herramienta de soldadura simulada configurada para ser rastreada espacialmente y una interfaz gráfica que permita al usuario realizar una o más entradas y permita ver la comparación entre el simulado y uno ideal; se resalta que con la Realidad Virtual se incurre en el ahorro de costos en general por el uso de herramientas y materiales poco comunes en los laboratorios, adicional a ello se evita exponer al 
estudiante a un riesgo que se puede mitigar con un adecuado entrenamiento, sin embargo la herramienta cuenta con una patente que restringe su uso de manera libre.

Por otro lado, Serrano [13] diseñó una aplicación de realidad virtual con escenarios 3D, apta para dispositivos de gama media-alta, que a través de una sala temática presenta diferentes desafíos que evalúan las capacidades del usuario y le ayudan en el aprendizaje sobre temas relacionados a la ciencia, dentro de los temas están son: el sistema solar a escala que permite interactuar con los diferentes planetas, ver los movimientos de la tierra y estudiar constelaciones, además, se pueden observar los huesos del cuerpo humano, ver dinosaurios y se pueden ver los elementos de la tabla periódica en 3D y al seleccionar uno de los elementos aparece un modelo 3D con los átomos, sin embargo, para usar la aplicación se requieren dispositivos de un costo elevado y solo está disponible para celulares.

Según Mitaritonna [14], el uso de la Realidad Aumentada en diferentes disciplinas se hace cada día más extensivo, no sólo por el potencial que brinda esta tecnología sino también por la capacidad que ofrece para mejorar los procesos cognitivos aplicados a la enseñanza, además indica que el uso de esta tecnología en la educación se debe centrar principalmente en activar los procesos cognitivos de aprendizaje. Por otra parte, Gopalan, et al. [15], proponen la implementación de libros de ciencias con Realidad Aumentada, para lograr un mayor interés en los estudiantes por las ciencias y su fácil aprendizaje, sin embargo, estos libros no están de manera gratuita en internet y tienen costo.

Ortega, Ortega \& Caballero [16] exponen cómo la Realidad Virtual está siendo potenciada por empresas como Google con su programa "Expeditions Pioneer Program", así mismo herramientas que permiten modificar los métodos de enseñanza tradicionales a través de Power Point, Keynote o Desk. ThingLin con las que se pueden generar entornos interactivos en los que se combinan contenidos en $3 \mathrm{D}$, vídeos, efectos de sonido, notas y presentaciones. Permitiendo abrir paso al programa E2H Education for Health, que busca la implementación de Realidad Virtual para intentar mejorar el proceso cardiovascular aumentando la velocidad de recuperación cardiaca a través de la proyección virtual que además trae consecuencias positivas a lo psíquico y emocional, aunque entre los aportes del artículo no se hace mención, al aprendizaje de química.

\section{Realidad Virtual en el Aprendizaje de Química.}

Alexiou, et al. [17] presentan como el uso de la Realidad Virtual puede disminuir los riesgos que representa un laboratorio, reducir costos de mantenimiento de herramientas y espacio y proveer a los estudiantes, un Entorno Virtual que permite generar una nueva experiencia de $\mathrm{e}$ learning, presentando un laboratorio de Radiofarmacia en 3D, sin embargo, está limitado a los estudiantes que se enfocaron en este tópico avanzado de Radiofarmacia.

Poblete [18], expone una nueva interfaz para el aprendizaje de la química para niños que están en la transición de la educación básica a la media, presentando una aplicación móvil llamada periodicAR que permite la interacción con elementos para obtener compuestos con la ayuda de Realidad Aumentada, en la cual se debe colocar un marcador del elemento que se quiere ver y al escanearlo muestra el modelo 3D del átomo (ver Figura 1). La herramienta solo está disponible en dispositivos móviles.

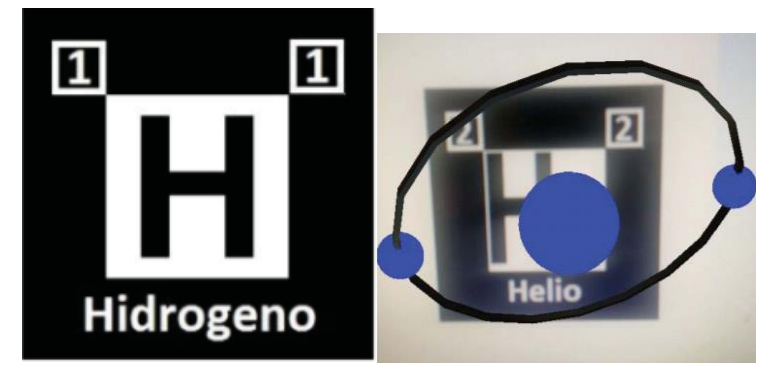

Figura 1. Imagenes de PeriodicAR, Poblete [9]

Herga, Čagran y Dinevski [19], presentan un laboratorio virtual para la enseñanza de química en estudiantes de 12 a 13 años que asisten a clases de ciencia; la investigación se limitó a dos temas 
de aprendizaje que son las propiedades y cambios de las sustancias y las sustancias y sus compuestos puros, se enfocan en la enseñanza de la química, pero no se centran en la tabla periódica.

Martínez, López, y Escalona [20] utilizan realidad aumentada para la creación de objetos 3D que se emplean como medios de enseñanza en el estudio de compuestos químicos como proteínas, medicamentos y estructuras cristalinas, pero no se hace mención de la enseñanza de la tabla periódica.

lordache, Pribeanu, y Balog [21] proponen una plataforma de enseñanza de química usando Realidad Aumentada, donde se proporciona una Tabla Periódica en la cual los estudiantes pueden colocar bolas de colores para completar las tareas. El investigador descubrió que la actividad de colocar bolas de colores sobre diferentes elementos químicos sobre la mesa les da a los niños la sensación de libertad y control, lo cual es beneficioso para su dominio, en este sentido, Romero [22], con su app Ocling-organic chemistry learning tiene como objetivo los estudiantes de primer semestre de universidad, buscando el fácil aprendizaje de la química orgánica. Esta app permite que su contenido sea configurado por los docentes, sin embargo la aplicación está limitada a un público objetivo.

Probst y Reymond [23] proponen una aplicación basada en el motor Unity y el kit de herramientas de realidad virtual (VRTK), que permite la exploración interactiva de un espacio químico poblado por compuestos del Banco de Drogas en realidad virtual. Así mismo, Hung et al., [24] desarrollaron modelos tridimensionales de compuestos de coordinación y emplearon realidad aumentada para integrarla con unos marcadores específicos, donde los estudiantes podían identificar, geometrías, tipos de isomería, y la disposición espacial de los orbitales de dichas geometrías, la herramienta la desarrollaron para la enseñanza de química en la facultad de ciencias naturales, de la universidad de Oriente y no está disponible para el público en general.
Bennie at al. [25] presentan un entorno virtual que permite interactuar a nivel molecular con los elementos, debido a que, este tipo de conceptos no se pueden ver a simple vista en los laboratorios, además este entorno permite evidenciar la acción de catalizadores en los compuestos, sin embargo se centra en un tema en específico y no en la Tabla Periódica.

\section{Realidad Virtual en el Aprendizaje de la Tabla Periódica.}

Yang, Mei y Yue [26], proponen una aplicación donde con ayuda de la cámara y unos marcadores específicos que la aplicación reconoce, se generan unos cubos transparentes con información específica de los elementos, como el nombre, símbolo, número atómico y peso atómico, además si dos elementos son colocados juntos la reacción química es simulada. Aguiña [27] usó una plataforma, donde construyeron unos marcadores que asociaron a unos modelos 3D, con el fin de que los estudiantes de primaria tuviesen una herramienta que los ayudara a entender, las reacciones químicas de la fermentación, de una manera más interactiva, debido a que estos son temas muy abstractos, sin embargo, tiene como público objetivo solo a estudiantes de primaria.

Lynch \& Ghergulescu [28] presentan un laboratorio virtual de química, donde los estudiantes están a cargo de su propio aprendizaje, en este, los estudiantes son participantes activos y no oyentes pasivos, el laboratorio virtual cuenta con tres niveles, que son átomos, isótopos y moléculas, el nivel inicial son los átomos y para avanzar deben responder preguntas hasta desbloquear el siguiente nivel; los estudiantes reciben comentarios instantáneos a medida que desarrollan los ejercicios y tienen la oportunidad de revisar lo que no aprendieron directamente después del ejercicio.

Taçgin, Uluçay \& Özüağ [29] proponen un escenario de aprendizaje de química basado en realidad aumentada donde los estudiantes pueden 
interactuar con la tabla periódica a través del movimiento de las manos y pueden seleccionar un elemento y ver la proyección del modelo atómico en 3D, además, estos pueden combinar los elementos y conseguir un compuesto químico. Presentan un entorno donde los estudiantes se enfrentan a situaciones problémicas y pueden poner en práctica sus conocimientos adquiridos, sin embargo, esta propuesta requiere de accesorios de alto costo para poder lograr la inmersión.

Setiawan, Rostianingsih y Widodo [30] proponen la construcción de una aplicación móvil que permite el aprendizaje de los elementos de la Tabla Periódica y las reacciones químicas utilizando Realidad Aumentada, la cual utiliza un marcador que la cámara reconoce y proyecta un objeto 3D del elemento químico (ver Figura 2). Los objetos interactúan entre sí al acercarse uno del otro, lo que permite la proyección de la reacción química de estos, pero la aplicación está limitada solo dispositivos móviles, además estos deben ser de una gama media-alta.

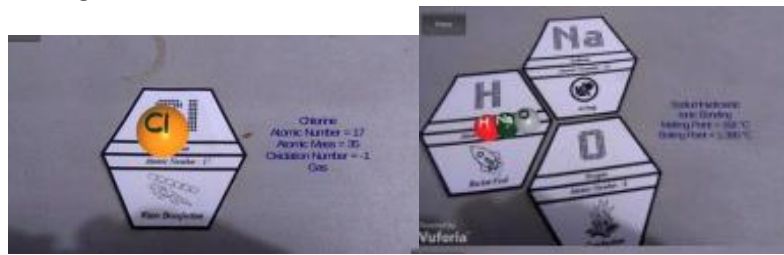

Figura 2. Proyección de elementos y reacción químicas [30]

Vargas, [31] expone la importancia de la tabla periódica en la química y evidencia la dificultad que tiene los estudiantes para aprenderla, esto debido a la falta de interés, siendo una de las razones que no se le da la verdadera importancia, además sugiere el uso de las TIC para promover el interés por aprender química de manera significativa de los estudiantes, sin embargo, no plantea usar tecnologías como la Realidad Virtual.

Luego de revisar las limitaciones encontradas en el estado del arte presentado, se puede concluir que es necesario una herramienta de apoyo para la enseñanza y aprendizaje que sea gratuita, multiplataforma que permite que cualquier persona con acceso a internet pueda usarla, sin necesidad de dispositivos adicionales de alto costo y procesamiento.

\section{MATERIALES Y MÉTODOS}

Inicialmente, se realizó la caracterización de los elementos de la tabla periódica, utilizando fuentes como la IUPAC (International Union of Pure and Applied Chemistry) [32], de la RSC (Royal Society of Chemistry) [33] y basados en la sugerencias de una docente universitaria de Química, posterior a ello, se implementaron los elementos más representativos de la Química orgánica o Química de la vida y finalmente se evaluaron algunas de las herramientas disponibles para la creación de entornos virtuales.

\section{Clasificación de los conceptos de la Tabla Periódica mediante la revisión de literatura y sugerencia de expertos.}

La distribución de los elementos de la Tabla Periódica (Ver Figura 3), fue presentada por primera vez por el químico Dmitri Mendeléiev; cada uno de los 118 elementos, está colocado a lo largo de filas o hileras, ordenadas de acuerdo a su número atómico creciente. Existen siete filas, cada una conforma un período. La longitud de los períodos se distribuye de la siguiente manera: el primero tiene dos elementos, los siguientes dos tienen ocho cada uno, luego 18 y 32 , respectivamente, en los siguientes pares de períodos. Las columnas verticales forman grupos, de los cuales hay 18, basados en propiedades químicas similares, relativas al número de electrones en la capa exterior de los átomos, también llamada capa de valencia [34].

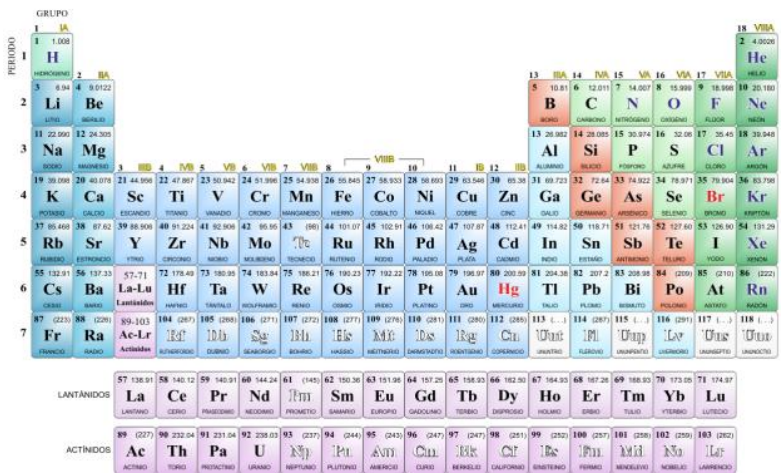

Figura 3. Tabla Periódica de los elementos [33] 
Los átomos de todos los elementos químicos están compuestos de partículas cargadas positivamente llamadas protones y son su número atómico (Ver Figura 4), un número igual de partículas cargadas negativamente llamadas electrones y partículas eléctricamente neutras llamadas neutrones. EI número total de protones y neutrones en un átomo específico [35].

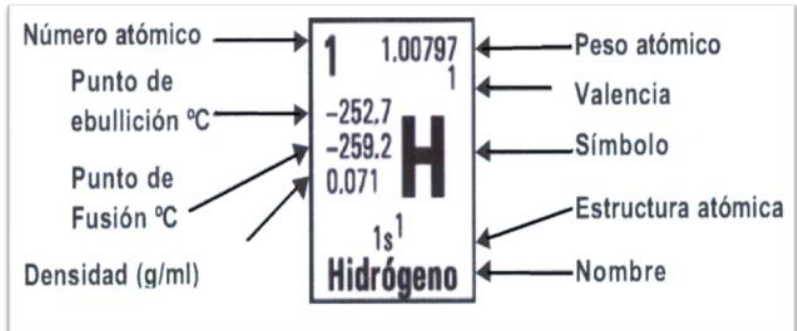

Figura 4. Elemento de la tabla periódica [35]

En el desarrollo de este entorno, se seleccionaron 18 elementos que son esenciales para la vida y se explica su función en ella, resaltando que se vive en simbiosis con otras bacterias, además, algunos elementos pueden ser fundamentales sólo en cantidades exactas; si se llega a presentar algún exceso, podría causar intoxicación o daños en el organismo, como el cobre, que ayuda al desarrollo de tejido conectivo, hueso y recubrimientos nerviosos, pero en grandes cantidades puede causar daño hepático [36]. Estos elementos, son los que se pueden seleccionar en el Entorno Virtual.

Los 18 elementos seleccionados fueron los siguientes: Oxígeno, Nitrógeno, Hidrógeno, Azufre, Fósforo, Potasio, Sodio, Cobalto, Selenio, Molibdeno, Cloro, Magnesio, Manganeso, Cobre, Zinc, Hierro, Calcio. De cada uno de ellos, se destacan los siguientes aspectos:

Nombre Elemento (Símbolo, Número Atómico (Z))

- En dónde se encuentra en la naturaleza o su abundancia natural.

- Su uso en la industria o su rol biológico, o ambos.

Dichos aspectos, se pueden observar en los siguientes ejemplos con el Hidrógeno y el Nitrógeno.

\section{Hidrógeno $(H, Z=1)$}

- Es el más abundante en el universo. Se encuentra en el sol y en la mayoría de las estrellas y el planeta Júpiter está compuesto principalmente de hidrógeno. Está presente en el agua y en casi todas las moléculas de los seres vivos.

- Es un elemento esencial para la vida. Sin embargo, el hidrógeno en sí no desempeña un papel particularmente activo. Permanece unido a los átomos de carbono y oxígeno, mientras que en la química de la vida tiene lugar en los elementos más activos que son, por ejemplo, oxígeno, nitrógeno y fósforo.

\section{Nitrógeno (N, Z=7)}

- El $78 \%$ del aire es nitrógeno, lo que lo convierte en el elemento más común en la atmósfera terrestre.

- El nitrógeno es ciclado naturalmente por los organismos vivos a través del "ciclo del nitrógeno", es absorbido por las plantas verdes y las algas como nitratos, y se utiliza para construir las bases necesarias de ADN, ARN y todos los aminoácidos. Los animales obtienen su nitrógeno consumiendo otros seres vivos. Digieren las proteínas y el ADN en sus bases constituyentes y aminoácidos, reformándolos para su propio uso.

Los elementos que participan y son fundamentales en la vida, se encuentran en su gran mayoría como compuestos y cada uno es importante desde su cantidad o concentración hasta su interacción con otros elementos, siendo esenciales para el correcto funcionamiento del cuerpo humano, como sistema digestivo u óseo y además la participación de ellos en la fotosíntesis que es la base de la vida.

Es por esto, que en el Entorno Virtual se muestra el elemento con su símbolo, nombre, peso atómico y número atómico, también, un modelo 3D relacionado, un cuadro donde se resalta la información más importante y un póster con información adicional de éste.

\section{Comparación de las diferentes herramientas relacionadas con realidad virtual.}

En esta sección, se compararon las diferentes características, ventajas y desventajas de herramientas que ayudan a la implementación de 
entornos virtuales, con el propósito de tener criterios para la elección de la herramienta que más se adecue a la creación del entorno virtual de la tabla periódica (ver Tabla 1).

Tabla 1 Comparación de las herramientas relacionadas con realidad virtual

\begin{tabular}{|c|c|c|c|c|c|c|}
\hline Herramienta & $\begin{array}{l}\text { Soft } \\
\text { ware } \\
\text { libre }\end{array}$ & $\begin{array}{l}\text { Orien } \\
\text { tada } \\
\text { a la } \\
\text { Web }\end{array}$ & $\begin{array}{c}\text { Sin } \\
\text { complem } \\
\text { entos } \\
\text { para la } \\
\text { Web }\end{array}$ & $\begin{array}{c}\text { Curva } \\
\text { de } \\
\text { aprendi } \\
\text { zaje }\end{array}$ & $\begin{array}{l}\text { Proyect } \\
\text { os } \\
\text { livianos }\end{array}$ & $\begin{array}{c}\text { Multiplata } \\
\text { forma }\end{array}$ \\
\hline Unity & Si & $\mathrm{Si}$ & No & Alta & No & $\mathrm{Si}$ \\
\hline $\begin{array}{l}\text { Unreal } \\
\text { Engine }\end{array}$ & No & $\mathrm{Si}$ & No & Alta & No & $\mathrm{Si}$ \\
\hline React 360 & Si & $\mathrm{Si}$ & $\mathrm{Si}$ & Baja & $\mathrm{Si}$ & $\mathrm{Si}$ \\
\hline Three.js & Si & $\mathrm{Si}$ & $\mathrm{Si}$ & Alta & Si & $\mathrm{Si}$ \\
\hline Janus $V r$ & $\mathrm{Si}$ & $\mathrm{Si}$ & No & Alta & $\mathrm{Si}$ & $\mathrm{Si}$ \\
\hline Blender & Si & $\mathrm{Si}$ & No & Baja & $\mathrm{Si}$ & $\mathrm{Si}$ \\
\hline SketchUp & No & No & No & Baja & $\mathrm{Si}$ & $\mathrm{Si}$ \\
\hline
\end{tabular}

Se tuvo en cuenta que el objetivo de la aplicación es que el estudiante pueda estar inmerso en un entorno virtual y que a su vez la herramienta sea asequible y de bajo costo y que no requiere ningún dispositivo con características especiales para lograr estar inmenso en el entorno de realidad virtual.

Basados en la caracterización de las herramientas se puede concluir que las que más se adecuan para la creación del entorno virtual son:

- React 360, debido a que esta está orientada a la Web y es multiplataforma, permitiendo que sea utilizada desde cualquier dispositivo, ya sea un televisor de aula de clase, un computador o el celular de los estudiantes, además, no requiere de licencias pagas, es fácil de utilizar y cumple con lo necesario para desarrollar el alcance de la investigación.

- Blender, permite modelar, renderizar, animar y crear gráficos en 3D de una manera ágil, es gratuita, es liviano, por lo cual no es necesario contar con equipos de alta gama para desarrollar en esta herramienta y su curva de aprendizaje es corta.

Por lo anterior, la combinación de estas dos herramientas permitió la implementación de un entorno virtual que ayude en la inmersión e interacción con los elementos anteriormente listados y caracterizados.

\section{Diseño del Entorno Virtual de la Tabla Periódica.}

En esta sección, se describe el diseño de la funcionalidad del software de forma general, tecnologías de desarrollo y técnicas de Realidad Virtual para la implementación del Entorno Virtual.

Se plantearon cuatro componentes que se listan a continuación para la proyección de los mockups.

- Como componente principal se propone un cuadro con la información básica del elemento, (ver Figura 5) la cual está basada en la descripción que se encuentra en la Tabla Periódica por cada elemento, seleccionando información como, peso atómico, símbolo, número atómico, entre otros.

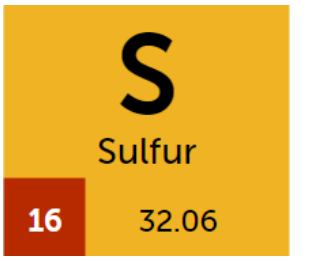

Figura 5. Elemento de la tabla periódica.

- Como segundo componente se implementó el modelado en tercera dimensión de objetos asociados al elemento (ver Figura 6). Esto con el objetivo de generar interacción en el entorno virtual y que se encuentre una relación entre la química y lo cotidiano. 


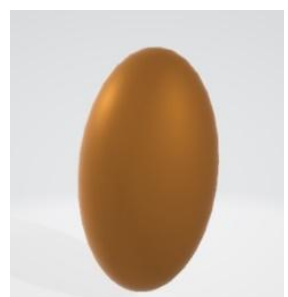

Figura 6. Modelo 3D asociado al azufre.

- En el tercer componente se agregó al entorno la información seleccionada en la caracterización (ver Figura 7).

\section{MAGNESIUM}

It is the eighth most abundant element in the earth's crust, but it does not occur without combining in nature. It is found in large deposits of minerals such as magnesite and dolomite. The sea contains billions of tons of magnesium, and this is the source of a large part of the 850,000 tons produced each year. It is the fourth most abundant element in the human body, present in the muscles and soft tissues (30-40\%), bones $60 \%$ and extracellular fluids $1 \%$.

Figura 7. Información caracterizada del elemento.

- En el cuarto y último componente se colocó el boletín que genera la IYPT (International Year of Periodic Table) donde se muestra información adicional al elemento (ver Figura 8).

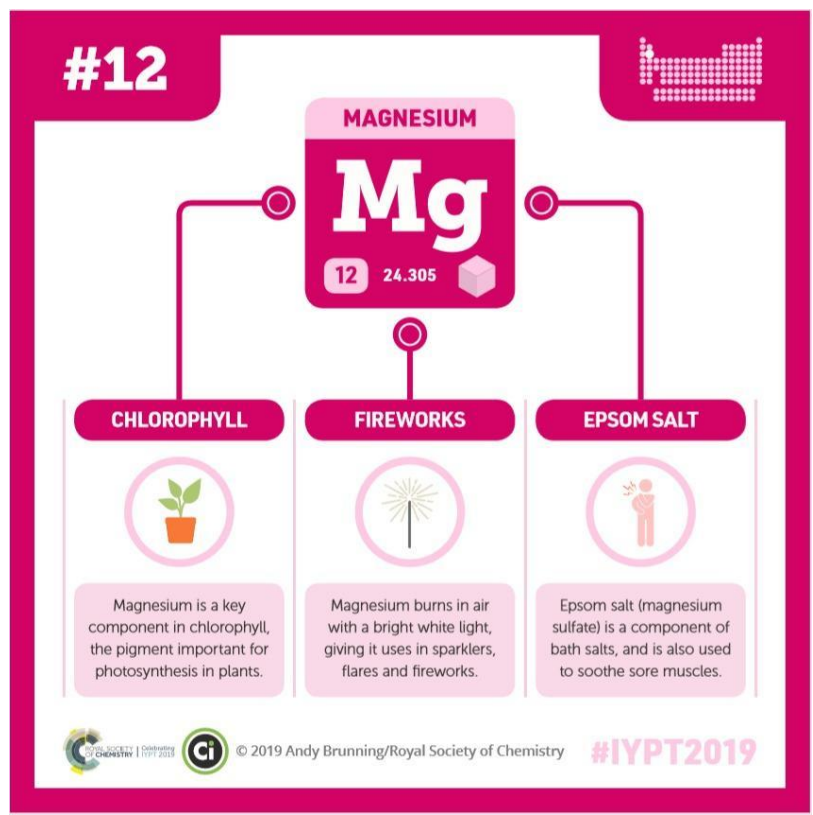

Figura 8. Boletín magnesio ( IYPT, 2019)

Posteriormente, se propone el diseño del entorno virtual en varios dispositivos y plataformas (ver Figura 9), con el propósito de proyectar una aplicación web que sea responsive o adaptativa, logrando que esta herramienta pueda ser usada por docentes desde un televisor, hasta por los estudiantes en sus dispositivos móviles, como celulares o tablets.
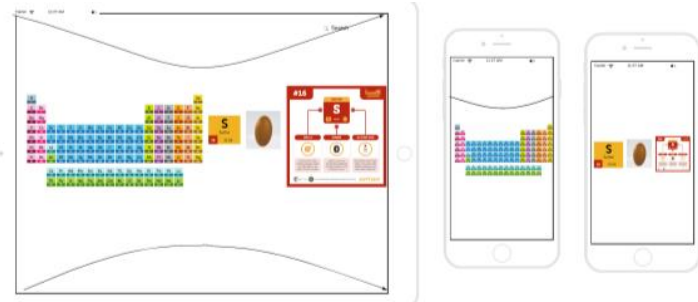

Figura 9. Mockups dispositivos

Para lograr el acople de los componentes y permitir a los estudiantes y docentes el uso del entorno virtual, primero, se planteó el patrón de diseño MVC (Modelo Vista Controlador) y tipo de arquitectura, en el cual se proyectaron las capas BackEnd, FrontEnd y los dispositivos a usar por un usuario final; segundo fue necesario la implementación de una base de dato, y como último se define el diagrama de flujo de la aplicación. El lenguaje que se usó en el BackEnd fue java y en el FrontEnd fue React 360. La herramienta que se utilizó para la realización de los diagramas fue LucidChart, esta permite realizar varios tipos de diagramas, entre ellos; UML, prototipos de software, esquemas de sitios WEB, entre otros.

- Patrón de diseño: La aplicación se desarrolló bajo el patrón de diseño Modelo Vista Controlador (MVC), donde el BackEnd es el encargado de controlar las peticiones a la Base de Datos y el FrontEnd mediante el SPA (Single Page Application) permite mostrar los elementos procesados en cualquier dispositivo(ver Figura 10). 


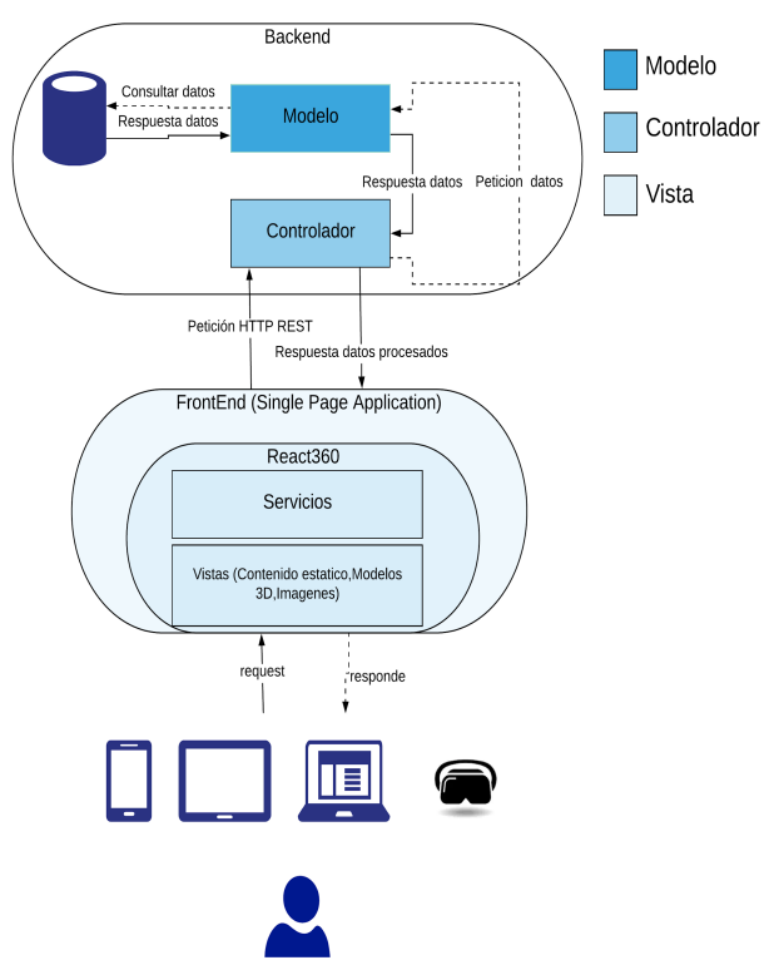

Figura 10. Arquitectura de la solución

- Base de datos(BD) : Se usará una BD relacional, debido a que, la información es consistente y no se planea mayor crecimiento superior a los 118 elementos, además, cubre las necesidades y el alcance del proyecto. Para la gestión de la BD se usó MySQL, herramienta de código abierto que es multiplataforma, con licencia no comercial gratuita y además no requiere de dispositivos con características especiales para brindar un adecuado rendimiento.

- Diagrama de Secuencia: En la Figura 11, se puede visualizar cómo interactúan los componentes y métodos cuando el usuario navega por el Entorno Virtual y se convierte en una petición al sistema.

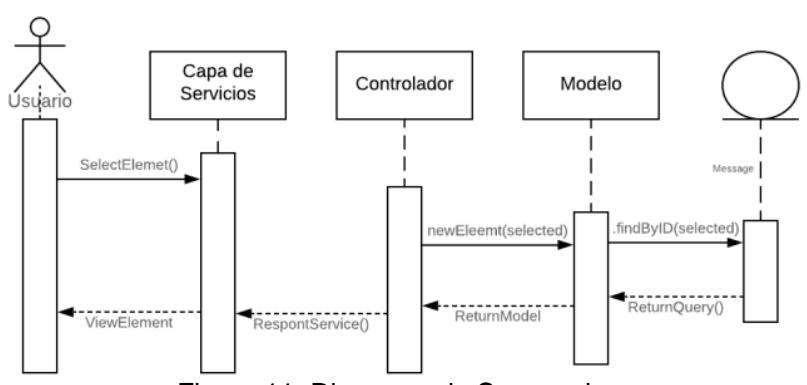

Figura 11. Diagrama de Secuencia.
Para la obtención del prototipo final, fue necesario permitir la comunicación completa entre todas las capas y componentes, desde la conexión a base de datos para recuperar la información del elemento e imágenes, hasta la capa de FrontEnd que se encarga de facilitar la interacción y hacer de la experiencia con el entorno virtual un momento inmersivo.

\section{EXPERIMENTOS Y RESULTADOS}

En esta sección se presenta el Entorno Virtual que se desarrolló basado en los diseños previamente realizados.

\section{Factores Técnicos.}

Se usaron las siguientes herramientas:

- MySQL version 8.0 como motor de base de datos.

- React 360 version 1.1.0 para el desarrollo del cliente del entorno virtual

- Blender version 2.79 para la realización de los modelos 3D

- Visual Studio como editor de código, en su versión 1.35.1

- GitHub para el control de versiones.

\section{Acople del entorno virtual}

Se implementó un Store basado en redux, el cual se encarga de la comunicación entre los diferentes componentes del entorno virtual. Por otro lado, se realizó una validación a través de funciones nativas de Javascript, para saber desde qué dispositivo se está accediendo; en caso de que este sea un teléfono celular o tenga un sensor de movimiento, se muestra un RayCaster, que es un puntero que aparece en la pantalla y sigue el movimiento del dispositivo, permitiendo la interacción con el Entorno Virtual y el uso de dispositivos o extensiones de Realidad Virtual tales como las Google Cardboard (ver Figura 12). 


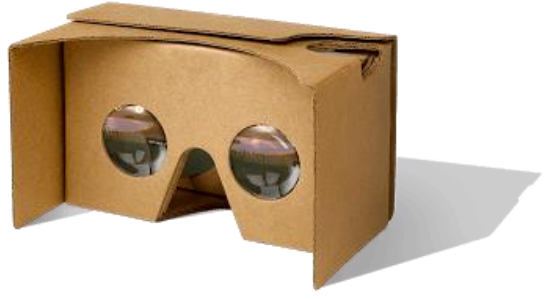

Figura 12. Google Cardboard

\section{Integración con Realidad Virtual.}

En este módulo se implementó un pequeño menú, donde se le indica al usuario cómo debe usar el Entorno Virtual dependiendo del dispositivo desde el cual esté accediendo, se tiene un botón para avanzar, uno para retroceder en el tutorial y otro en la parte superior para cerrarlo, al final del tutorial se presenta un ejemplo (ver Figura 13)

\section{Implementación del Tutorial.}

En este módulo se implementó un pequeño menú, donde se le indica al usuario cómo debe usar el Entorno Virtual dependiendo del dispositivo desde el cual esté accediendo, se tiene un botón para avanzar, uno para retroceder en el tutorial y otro en la parte superior para cerrarlo, al final del tutorial se presenta un ejemplo (ver Figura 13)

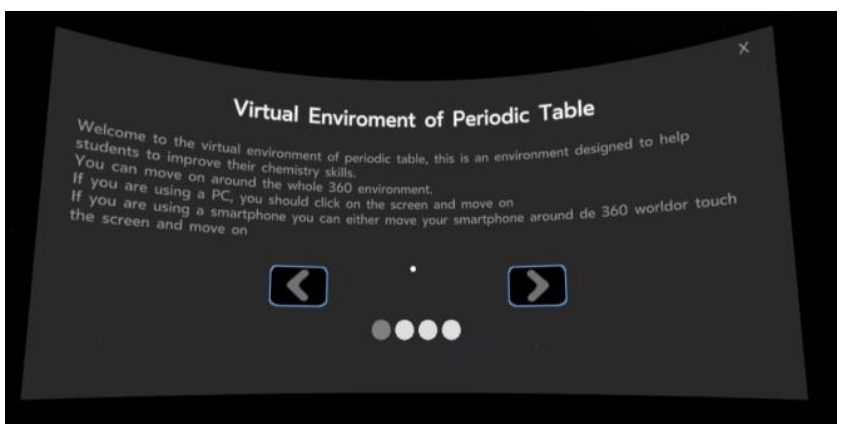

Figura 13. Vista del tutorial

\section{Implementación de la Tabla Periódica}

Para la construcción de la tabla periódica, se tuvieron en cuenta las sigues funcionalidades:

- Se obtienen todos los elementos de la base de datos.
- Se realiza la validación de cuales elementos están activos en el entorno virtual.

- Se ordenan los elementos por grupo.

- Se realiza un ciclo en el cual, por cada grupo se construye un contenedor donde la longitud del contenedor depende del número de elementos que contenga el grupo y se colocan todos los elementos de este, hasta completar toda la Tabla Periódica dejando con un brillo más alto los elementos que están activos en el entorno virtual (ver Figura 14).

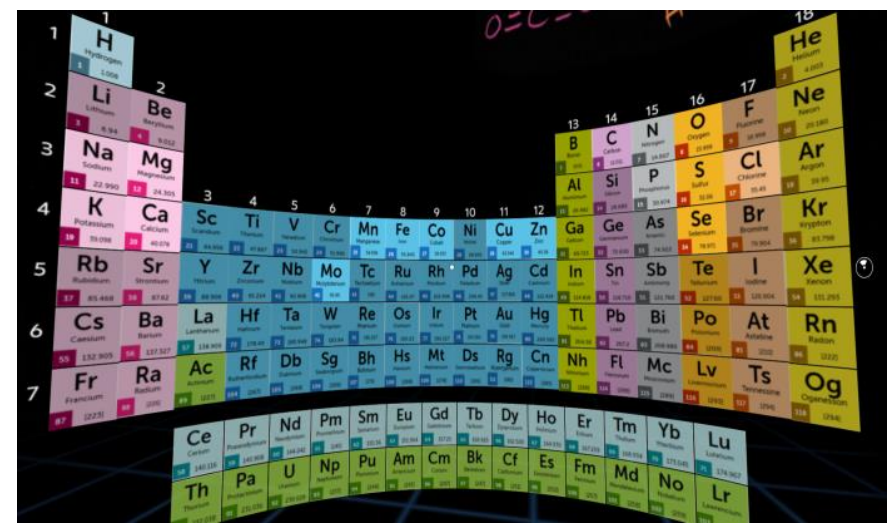

Figura 14. Vista de la tabla periódica

- Este componente, siempre está interactuando con los demás componentes del Entorno Virtual. Cada elemento de la tabla periódica tiene asociado el ID, al presionar click sobre este, se le envía al Store cuál elemento fue el seleccionado y este se encarga de comunicarse con el resto de componentes.

\section{Implementación de la Información Caracterizada.}

Este módulo se dividió en 4 componentes, donde cada uno de estos, está suscrito al Store basado en redux, para que cuando se seleccione un elemento en el componente de la tabla periódica, se emita un evento y éstos puedan obtener qué elemento se seleccionó y la información de este; además, tienen una validación para que cuando no se haya seleccionado ningún elemento o la aplicación se encuentre en el tutorial, no muestre ninguna información. Los componentes implementados son: 
- El componente con la información del elemento ampliada, tal como nombre, símbolo, número atómico y peso atómico.

- $\quad$ El componente donde se muestra un modelo $3 \mathrm{D}$ relacionado al elemento seleccionado (ver Figura 15); para este componente se creó un escenario, en el cual se coloca la iluminación y se define la posición del modelo 3D. Posterior a esto, se carga el modelo al Entorno Virtual dependiendo del seleccionado en la Tabla Periódica. En este componente, se dejan variables paramétricas configurables desde la base de datos, tales como el tamaño en sus tres coordenadas $\mathrm{x}, \mathrm{y}, \mathrm{z}$ y la dirección de rotación del elemento.

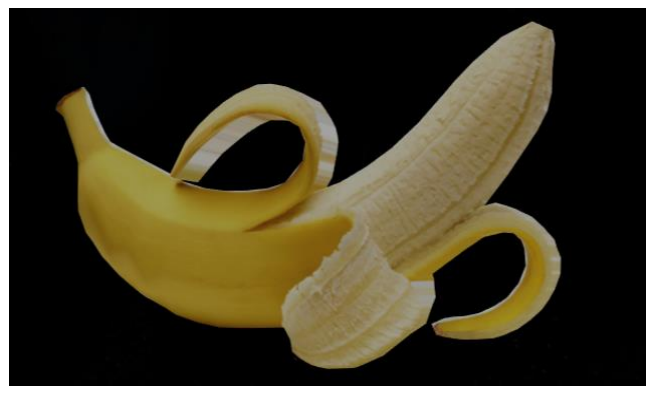

Figura 15. Modelo 3D

- El componente con la información caracterizada, es una vista, donde se coloca el texto en inglés, de la información relacionada al elemento seleccionado. Al pasar el mouse o el rayCaster sobre este, se amplia para facilitar la lectura.

- Se implementa el componente donde se coloca la imagen sacada de la IYPT relacionada al elemento seleccionado, y en este mismo se coloca un botón que redirecciona a la encuesta.

La validación se realizó con diferentes personas que se clasificaron según su rol en: Estudiantes, docentes, desarrolladores $u$ otro (personas allegadas). También, se diseñó una encuesta para medir la percepción acerca de la funcionalidad del software y la satisfacción, respecto al propósito de la aplicación. Con base en lo anterior, se siguieron los siguientes pasos:

Primero se eligió un grupo de estudiantes $(38 \%$ de la muestra) de la asignatura Química Orgánica del
Politécnico JIC. Se les hizo una introducción sobre el Entorno Virtual y cómo pueden usarlo de acuerdo al dispositivo en el que se encontraban, entre ellos, tablet, proyección en televisor, Google Cardboard o computador, posterior a ello, se les invitó a interactuar con el Entorno Virtual. Esto mismo se realizó con personas allegadas, profesores $(6 \%)$ y desarrolladores de un banco de Medellín (44\%) y por último, el grupo de personas allegadas que no cumplían ninguno de los roles anteriormente mencionados (12\%).

Al finalizar la interacción con la aplicación se les solicitó a las personas diligenciar una encuesta. Entre los aspectos evaluados por las personas que realizaron la validación, la mayoría de las personas afirmaban sentirse inmersos en la aplicación (ver Figura 16).

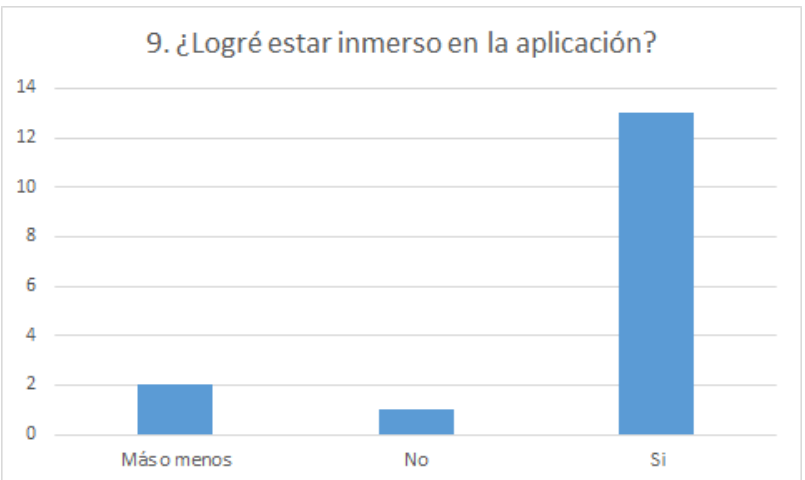

Figura 16. Percepción de inmersión de los usuarios.

En cuanto al uso de la herramienta para el aprendizaje, la mayoría de las personas están interesadas en usar la aplicación, para facilitar y mejorar la experiencia de aprendizaje (ver Figura 17).

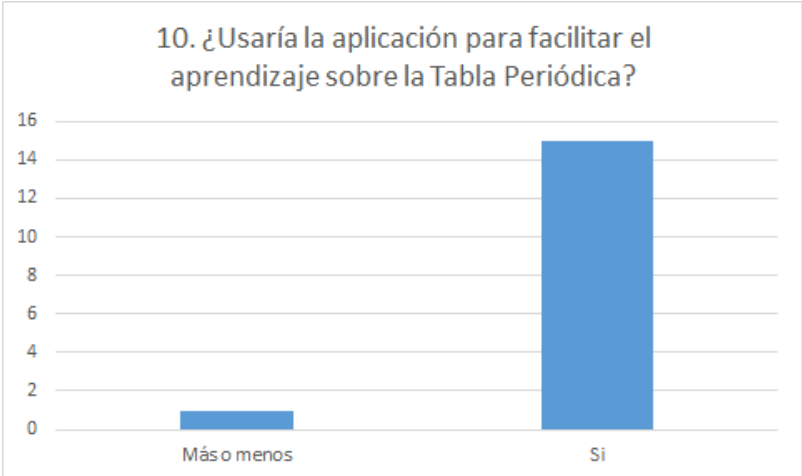

Figura 17. Tendencia de uso para facilitar el aprendizaje. 
Entre los aspectos a mejorar que sugieren los usuarios se encuentra, el correcto funcionamiento de la aplicación en todos los dispositivos, debido a que, durante la validación se evidenció que el modo de Realidad Virtual no estaba disponible en todos los dispositivos, también entre las sugerencias más comunes está la calidad de los gráficos que mejore tipo y tamaño de letra cuando se usan las cardboard y disminuir la vibración del entorno en general. Esto se atribuye al bajo costo de las cardboard, ya que en dispositivos como computadores o tablets no se evidencia esto.

Para finalizar, lo positivo que resaltan los usuarios es que encontraron información de su interés de la Tabla Periódica, además que se muestran objetos asociados a su cotidianidad que les permitió conocer nuevas características de los elementos de la Tabla Periódica, también resaltan que pueden ver moléculas, les gustó el efecto $3 D$ que se permite una inmersión e interacción con en el entorno, debido a que, esto permite mantener la atención de quien aprende y cuando se usan las gafas solo es necesario usar el puntero lo que facilita la interacción y por último se rescata entre los comentarios que es algo novedoso.

Después de evaluar lo evidenciado en la validación, se diagnosticaron las dificultades presentadas para mitigarlas o solucionarlas en su totalidad, de igual manera se desarrolla un manual de usuario para la configuración en dispositivos móviles, lo cual fue uno de los aspectos en el que se presentaron mayores dificultades; también se realizó la adecuación de la forma en la que se seleccionan los elementos con las gafas de Realidad Virtual y se modificó el tutorial para que fuera más intuitivo debido a que las personas no sabían cómo salir de este.

Se resalta la importancia de este capítulo debido a que se evidenció que se alcanzó el objetivo de generar una nueva herramienta que los estudiantes estén interesados en utilizar y que la interacción con el Entorno Virtual si les aporte nuevos conocimientos. Adicional a esto se plantean algunas mejoras obtenidas en la validación, como permitir que el Entorno Virtual sea más intuitivo y no requiera de una introducción o del tutorial para lograr la mejor experiencia con la aplicación.

\section{CONCLUSIONES Y TRABAJOS FUTUROS}

La dificultad para aprender Química va relacionada con los conceptos abstractos que pueden ser difíciles de comprender a la hora de ser expuestos por los libros o la explicación del docente. Dado esto, se infiere que las TIC deben de ser implementadas en la educación no como un fin, sino, como el medio para lograr la motivación, interés y desarrollo del correcto conocimiento por la Química.

Con el desarrollo del Entorno Virtual se logró la implementación de la Tabla Periódica, para ser usada como un Entorno Educativo, que después de la validación fue posible evidenciar que a través de este es posible hacer una relación entre lo real y práctico en un solo momento, logrando nuevas experiencias relacionando objetos cotidianos a los elementos de la Tabla Periódica que faciliten la recordación.

Como trabajos futuros se propone la continuación del desarrollo de los elementos faltantes de la Tabla Periódica, ampliar información que se muestra, por ejemplo, agregar el modelo atómico de cada elemento, generar un espacio para describir a quienes descubrieron el elemento, de igual manera hallar la solución al issue encontrado en la librería de React, que no permite acceder al giroscopio de los dispositivos sin la modalidad de RV. Este issue se documentó en la comunidad de React, a la fecha se tiene un comentario por parte de uno de los miembros, que afirma tener el mismo error, pero aún no hay solución a ello.

\section{AGRADECIMIENTOS.}

A la profesora Claudia Sánchez, por el apoyo en el desarrollo de este proyecto, por compartirnos sus conocimientos, por su tiempo y dedicación.

\section{REFERENCIAS BIBLIOGRÁFICAS}

\section{Artículos:}

[1] Martín-Gutiérrez, J., Mora, C. E., Añorbe-Díaz, B., \& González-Marrero, A. Virtual technologies trends in education. EURASIA Journal of Mathematics Science and Technology Education, 13(2), 469-486, $2017 .$. 
[3] Cai S., Wang, X., \& Chiang, F. K. A case study of Augmented Reality simulation system application in a chemistry course. Computers in human behavior, 37, 31-40, 2014.

[4] Torres Nieves, F. Laboratorios virtuales como estrategia para la enseñanza de la química, 2017.

[5] ROEHL, B. Special edition using VRML. Mc Millan Computer Publishers, 1996.

[9] Lasch, P. La mesa de juego de Mendeleiev, Ciencias, 65, 76, 2002.

[10] El-Mounayri, H. A., Rogers, C., Fernandez, E., \& Satterwhite, J. C. (2016). Assessment of STEM e-Learning in an immersive virtual reality (VR) environment. American Society for Engineering Education.

[11] Zhang, K., Suo, J., Chen, J., Liu, X., \& Gao, L. (2017, September). Design and implementation of fire safety education system on campus based on virtual reality technology. In 2017 Federated Conference on Computer Science and Information Systems (FedCSIS) (pp. 1297-1300). IEEE.

[12] Peters, C., Postlethwaite, D., \& Wallace, M. W. (2016). U.S. Patent No. 9,280,913. Washington, DC: U.S. Patent and Trademark Office.

[13] Sánchez Serrano, E. (2018). Aplicación en realidad virtual sobre distintos juegos con fines educativos.

[14] Mitaritonna, A. D. (2018). Tecnologías emergentes en la educación: la realidad aumentada. Perspectivas: Revista Científica de la Universidad de Belgrano, 1(2), 85-93.

[15] Valarmathie Gopalan, A. N. Z., Mohamed, N. F. F., Alwi, A., Mat, R. C., Bakar, J. A. A., \& Saidin, A. Z. (2016). Augmented Reality Books For Science Learning-A Brief Review.

[16] Ortega, M., Ortega, I., \& Caballero, A. O. (2018). Educación y salud. La realidad virtual como agente. TRANCES. Transmisión del Conocimiento Educativo y de la Salud, (1), 439-458.
[17] Alexiou, A., Bouras, C., Giannaka, E., Kapoulas, V., Nani, M., \& Tsiatsos, T. (2004, March). Using VR technology to support e-learning: the $3 \mathrm{D}$ virtual radiopharmacy laboratory. In 24th International Conference on Distributed Computing Systems Workshops, 2004. Proceedings. (pp. 268273). IEEE.

[19] Herga, N. R., Čagran, B., \& Dinevski, D. (2016). Virtual laboratory in the role of dynamic visualisation for better understanding of chemistry in primary school. Eurasia Journal of Mathematics, Science \& Technology Education, 12(3), 593-608.

[20] Martínez-Hung, H., García-López, A., \& Escalona-Arranz, J. C. (2017). Modelos de Realidad Aumentada aplicados a la enseñanza de la Química en el nivel universitario. Revista Cubana de Química, 29(1), 13-25.

[21] lordache, D. D., Pribeanu, C., \& Balog, A. (2012). Influence of specific AR capabilities on the learning effectiveness and efficiency. Studies in Informatics and Control, 21(3), 233-240.

[23] PROBST, Daniel; REYMOND, Jean-Louis. Exploring drugbank in virtual reality chemical space. Journal of chemical information and modeling, 2018, vol. 58, no 9, p. 1731-1735.

[24] Hung, H. M., López, A. G., González, O. Q., \& Verdecias, I. A. (2019). Realidad aumentada en la enseñanza de la química de coordinación y estructura de sólidos. Atenas, 2(46), 111-125.

[25] Bennie, S., Ranaghan, K., Deeks, H., Goldsmith, H., O'Connor, M., Mulholland, A., \& Glowacki, D. (2019). Teaching enzyme catalysis using an open source framework for interactive molecular dynamics in virtual reality.

[26] Yang, S., Mei, B., \& Yue, X. (2018). Mobile Augmented Reality Assisted Chemical Education: Insights from Elements 4D. 
[27] Aguiñiga, P. G. (2016). Realidad aumentada para enseñar Química en Educación Primaria: La fermentación.

[28] Lynch, T., \& Ghergulescu, I. (2018, June). Innovative pedagogies and personalisation in STEM education with NEWTON Atomic Structure Virtual Lab. In EdMedia+ Innovate Learning (pp. 1483-1491). Association for the Advancement of Computing in Education (AACE).

[29] TAÇGIN, Z., ULUÇAY, N., \& ÖZÜAĞ, E. (2016). Designing and Developing an Augmented Reality Application: A Sample of Chemistry Education. Turkiye Kimya Dernegi Dergisi, Kisim C: Kimya Egitimi, 1(1), 147-164

[30] Setiawan, A., Rostianingsih, S., \& Widodo, T. R. (2018). Implementation of Chemical Reaction Based on Augmented Reality. Advances In Natural And Applied Sciences, 12(4), 22-26.

[31] Tuarez Vargas, R. A. (2018). El manejo de una tabla periódica interactiva en el proceso de aprendizaje de química, de los estudiantes de primero Bachillerato General Unificado de la Unidad Educativa 11 de marzo del cantón Quito, Provincia de Pichincha, en el año Lectivo 20162017 (Bachelor's thesis, Universidad de Guayaquil, Facultad de Filosofía, Letras y Ciencias de la Educación).

[34] Scerri, E. El pasado y el futuro de la tabla periódica: Este fiel símbolo del campo de la química siempre encara el escrutinio y el debate. educación química, 19(3), 234-241, 2008.

[35] Norman E. Holden, Tyler B. Coplen, John K. Böhlke, Lauren V. Tarbox, Jacqueline Benefield, John R. de Laetera, Peter G. Mahaffy, Glenda O'Connorb, Etienne Rotha, Dorothy H. Tepper, Thomas Walczyk, Michael E. Wieser and Shigekazu Yoneda. IUPAC Periodic Table of the Elements and Isotopes (IPTEI) for the Education Community (IUPAC Technical Report), 2018.
[36] CHEN, Pan; BORNHORST, Julia; ASCHNER, Michael. Manganese metabolism in humans. 2019.

\section{Libros:}

[2] Brown, T. L., LeMay Jr, H. E., Bursten, B. E., \& Burdge, J. R. Química. Pearson Educación. 2004

[6] Su, C. H., \& Cheng, T. W. A Sustainability Innovation Experiential Learning Model for Virtual Reality Chemistry Laboratory: An Empirical Study with PLS-SEM and IPMA. Sustainability, 11(4), 1027, 2019.

[7] Jerald, J. The VR book: Human-centered design for virtual reality. Morgan \& Claypool, 2015.

\section{Tesis:}

[8] Cárdenas, S., \& Cumandá, N. (2015). Desarrollo de un entorno virtual que facilite la decodificación de textos escritos en estudiantes con problemas de lectura (Master's thesis, Pontificia Universidad Católica del Ecuador Sede Ambato.

[18] Poblete, E. M. A. Aprendizaje de la química con realidad aumentada (Doctoral dissertation, pontificia universidad católica de Valparaíso, 2016.

[22] Melo Romero, D. O. (2016). Ocling-organic chemistry learning: aplicación para apoyar la enseñanza de química orgánica en universidades (Bachelor's thesis, Facultad de Ingeniería).

\section{Referencias de internet:}

[32] International Union of Pure and Applied Chemistry. Periodic Table of Elements. [página Web] (Dic). Disponible en internet: https://iupac.org/what-we-do/periodic-table-ofelements/

[33] Royal Society of Chemistry. Periodic Table [página Web]. [Consultado: 21 de mayo de 2019]. Disponible en internet: http://www.rsc.org/periodictable. 